\title{
RANGELAND GRAZING AS A SOURCE OF STEROID HORMONES TO SURFACE WATERS
}

\author{
Supporting Information \\ Submitted to: \\ Environmental Science and Technology \\ December 21, 2006 \\ Revised March 5, 2007 \\ Edward P. Kolodziej ${ }^{\dagger}$ \\ David L. Sedlak* \\ Department of Civil and Environmental Engineering \\ 657 Davis Hall \\ University of California \\ Berkeley, CA 94720
}

*Corresponding author: Telephone (510) 643-0256; Fax (510) 642-7483; email:

sedlak@ce.berkeley.edu

${ }^{\dagger}$ Current address: Department of Civil and Environmental Engineering, University of Nevada, Reno, Reno, NV 89557 
Chemical Analysis Summary. Steroid hormones were extracted using C-18 solid phase extraction discs followed by derivatization and gas chromatography-tandem mass spectrometry (GC/MS/MS). Suspended particles were removed by pressure-filtering 4-L aliquots of sample through 90-mm AP 40 glass-fiber filters (Fisher Scientific, Pittsburg, PA). The surrogate standards $d_{5}-17 \beta$-estradiol, $d_{4}$-estrone, and mesterolone were then spiked into the filtered samples to a concentration of $100 \mathrm{ng} / \mathrm{L}$. Steroid hormones were pressure-extracted from the filtrate using pre-conditioned 90-mm Empore (3-M, Minneapolis, MN) C-18 solid-phase extraction discs. To remove polar organic matter from the extraction discs, the C-18 discs were washed twice with $25 \mathrm{~mL}$ of a 75:25 water:methanol solution prior to elution of the steroid analytes with $20 \mathrm{~mL}$ of a 10:90 water:methanol solution. The eluent was dried under vacuum, resuspended in methanol, and transferred to flasks. After again drying under vacuum, the extract was resuspended in $200 \mu \mathrm{L}$ HPLC-grade acetonitrile and derivatized with $50 \mu \mathrm{L}$ heptafluorobutyric anhydride, sealed, and placed in a $55^{\circ} \mathrm{C}$ oven for $1.5 \mathrm{hrs}$. The samples were then cooled and evaporated under a gentle stream of nitrogen prior to resuspension in $100 \mu \mathrm{L}$ of iso-octane. The overall concentration factor for the sample extracts during this procedure was 40,000 fold.

Relevant analytical conditions, GC/MS/MS instrument parameters, and method detection limits for the steroid hormones are summarized in Table S1. Positive identification of steroids was based upon retention time and MS/MS daughter ion abundance ratios. For analyte identification and quantification, retention times for the analytes had to match retention times of reference compounds within $0.1 \mathrm{~min}$. Also, the abundance ratios of the MS/MS daughter ions had to match the abundance ratios of the reference compounds within $20 \%$. Calibration was performed with linear 7-point curves from $1.0 \mu \mathrm{g} / \mathrm{L}$ to $100 \mu \mathrm{g} / \mathrm{L}$, with points equally spaced on a 
logarithmic scale. The limit of quantification was based upon the lowest calibration point of the calibration curve (i.e., 0.3 or $0.4 \mathrm{ng} / \mathrm{L}$ after accounting for sample pre-concentration). The method limit of detection is approximately $1 / 3$ of the limit of quantification (i.e., approximately $0.1 \mathrm{ng} / \mathrm{L}$ ). Additionally, to be considered for quantification, the signal-to-noise ratios for the analytes needed to exceed 6. Quantification was accomplished using the summed areas of the MS/MS base peak ion and any confirmatory qualifier ions. Peak areas were normalized to the surrogate standard area counts to correct for variations in derivatization efficiency, analyte recovery, and GC/MS/MS performance.

An additional objective of this study was to determine whether easily measured surrogate water quality parameters such as nitrate, total coliforms, and $E$. coli were predictive for steroid occurrence and concentration. Nitrate was analyzed by ion chromatography using a Dionex DX120 ion chromatograph equipped with an IonPac AS-14 column and an AS-40 autosampler. Coliforms were measured using the Colilert assay (IDEXX, Westbrook, ME) and coliform concentrations were quantified using the Quanti-Tray/2000 (IDEXX) and the Most Probable Number (MPN) model. 

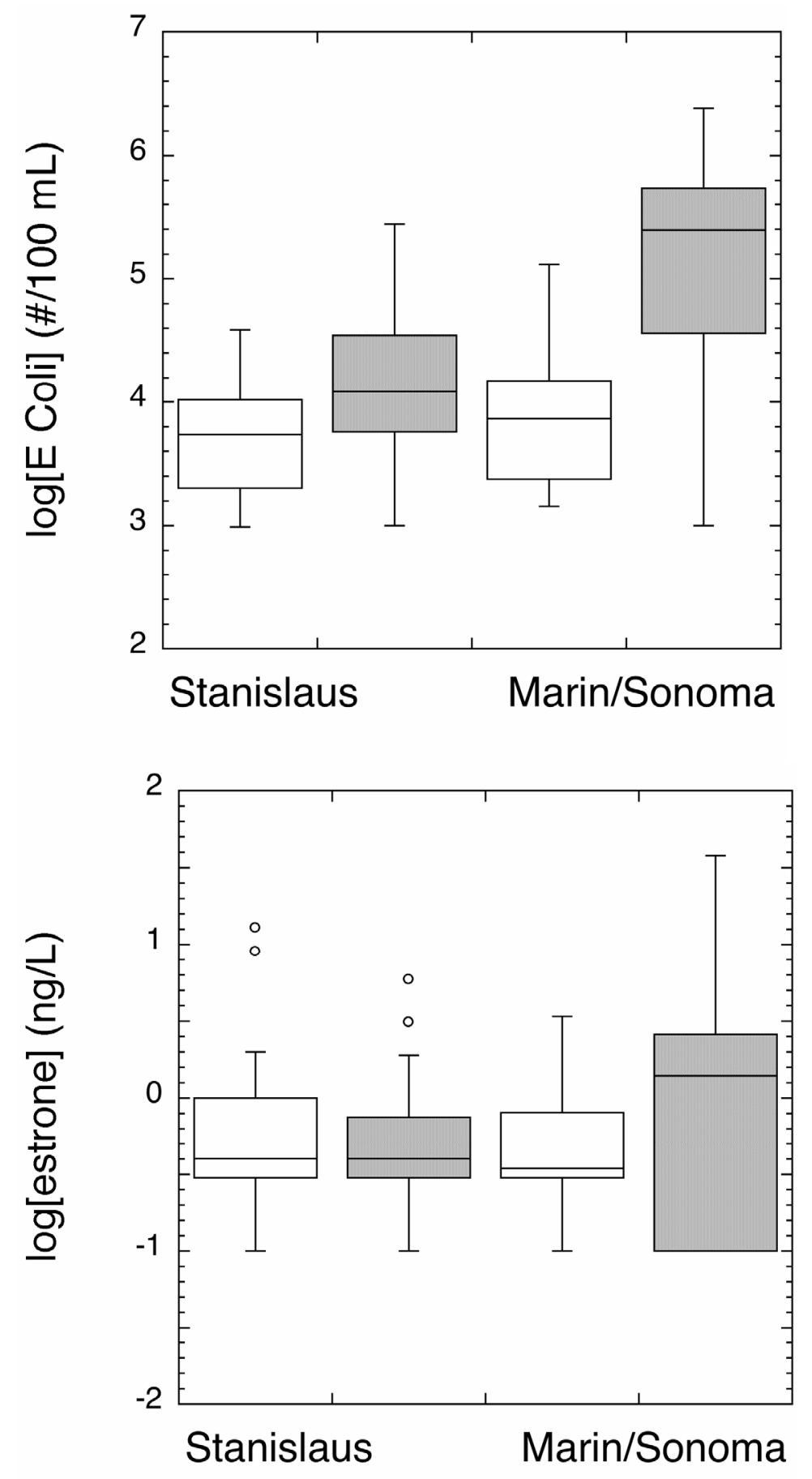

Figure S1: Box and whisker plots for log-transformed data on E. Coli (top) and estrone (bottom) during dry (unshaded) and wet (shaded) seasons. 


\begin{tabular}{|c|c|c|c|c|c|c|c|c|c|c|c|}
\hline 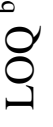 & $\begin{array}{l}\underset{\infty}{\overrightarrow{0}} \\
\underset{E}{ }\end{array}$ & $\ddot{0}$ & $\stackrel{+}{\circ}$ & $\begin{array}{l}0 \\
0\end{array}$ & ma & $\stackrel{m}{0}$ & $\ddot{\infty}$ & $\stackrel{m}{0}$ & $\stackrel{+}{\circ}$ & $\stackrel{\nabla}{\circ}$ & $\stackrel{\nabla}{\circ}$ \\
\hline$\stackrel{\sigma}{\overbrace{-}}$ & $\underset{\infty}{\underset{\Xi}{\Xi}}$ & $\stackrel{-}{0}$ & $\stackrel{+}{\stackrel{7}{-1}}$ & 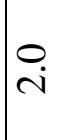 & 뭉 & $\stackrel{-}{0}$ & $\ddot{\circ}$ & $\stackrel{-1}{0}$ & $\stackrel{\Xi}{\stackrel{\Xi}{0}}$ & $\stackrel{\Xi}{\rightleftarrows}$ & $\stackrel{\vec{Z}}{\stackrel{ \pm}{0}}$ \\
\hline 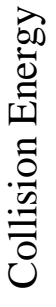 & 主 & $\stackrel{\overbrace{}}{\stackrel{\overbrace{}}{\overbrace{}}}$ & - & $\mathbb{Z}$ & ָָ & $\stackrel{0}{ }$ & $\stackrel{\overbrace{}}{\stackrel{一}{\sim}}$ & بـ & ن. & 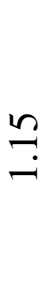 & 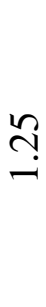 \\
\hline 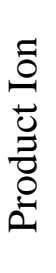 & 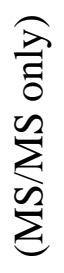 & $\begin{array}{l}0 \\
\text { ஸे }\end{array}$ & 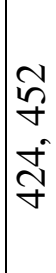 & 艺 & ฟै & 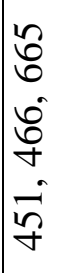 & 芶 & 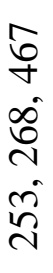 & 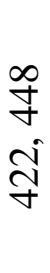 & $\begin{array}{l}\mathscr{\infty} \\
\text { m }\end{array}$ & 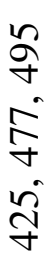 \\
\hline 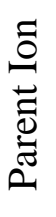 & & ஜ) & $\overrightarrow{\widehat{\gamma}}$ & 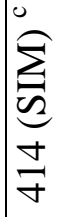 & ర్ర & Dేర & ర్ & 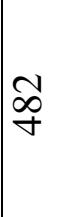 & $\hat{\vartheta}$ & $\underset{\sim}{\stackrel{R}{+}}$ & $\overrightarrow{\overline{1}}$ \\
\hline 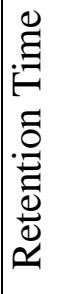 & 当 & $\begin{array}{l}\text { 오 } \\
\text { in } \\
\dot{I}\end{array}$ & $\begin{array}{l}\text { Oे } \\
\text { ம் }\end{array}$ & $\begin{array}{l}\text { ○ } \\
\infty \\
\infty\end{array}$ & $\begin{array}{l}\stackrel{\rho}{m} \\
\ddot{m} \\
\end{array}$ & $\begin{array}{l}\text { 늠 } \\
\stackrel{ \pm}{ \pm}\end{array}$ & $\begin{array}{l}\text { ஸे } \\
\dot{J} \\
\end{array}$ & $\begin{array}{l}\text { லூ } \\
\text { ம் }\end{array}$ & $\begin{array}{l}8 \\
0 \\
0\end{array}$ & $\begin{array}{l}\text { సి } \\
\text { बे }\end{array}$ & ڤُ \\
\hline 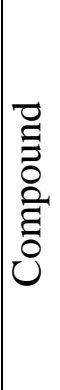 & & 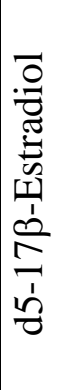 & 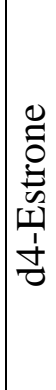 & 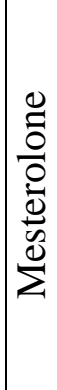 & 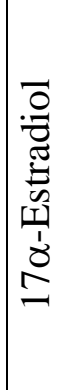 & 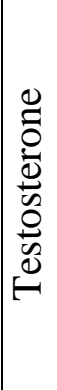 & 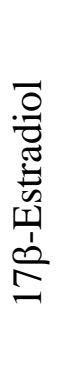 & 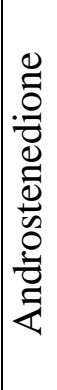 & 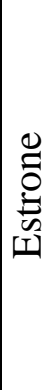 & 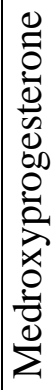 & 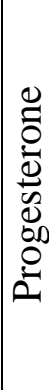 \\
\hline
\end{tabular}




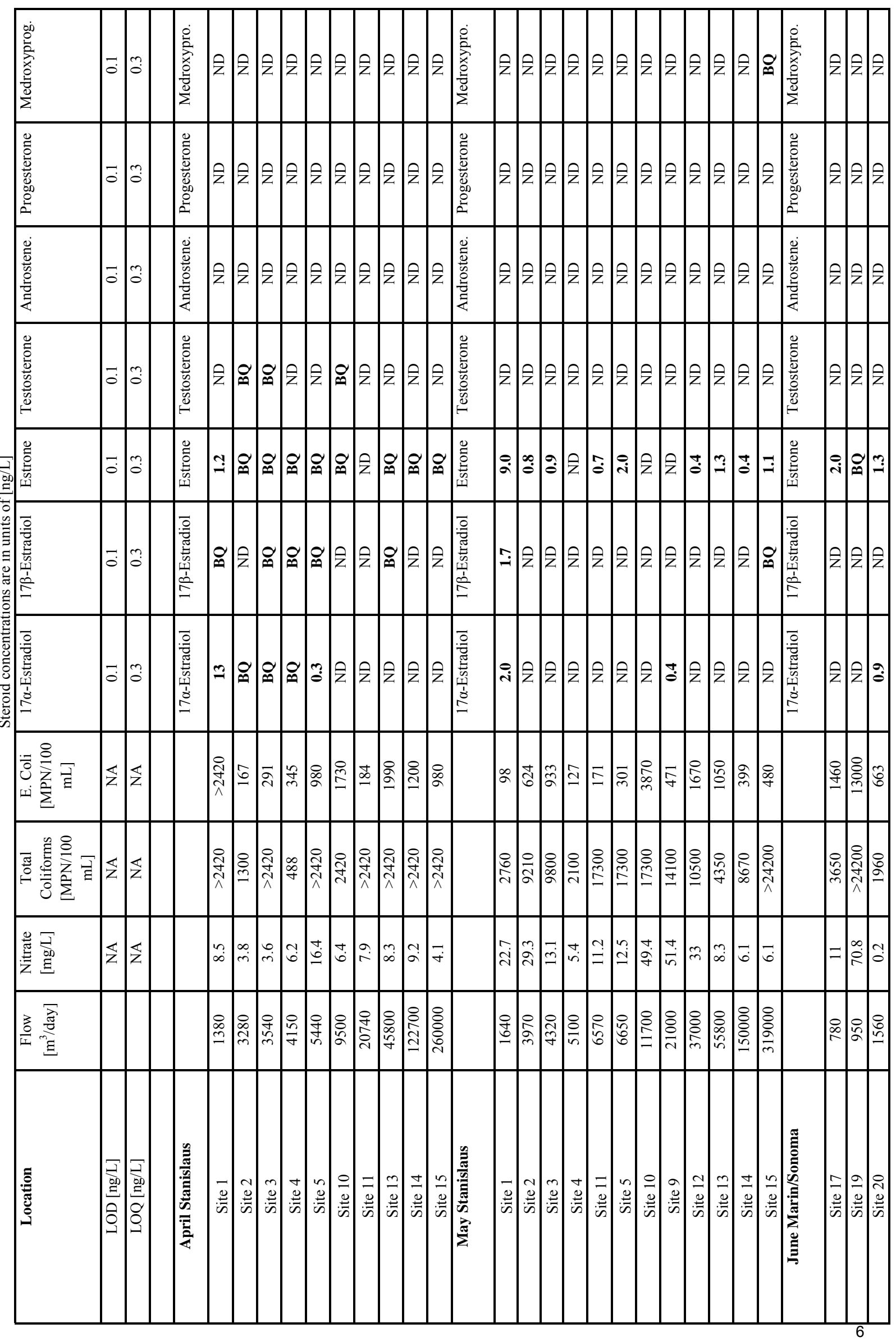




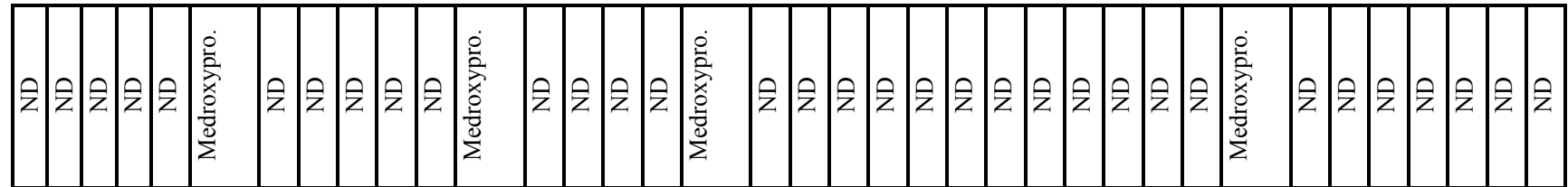

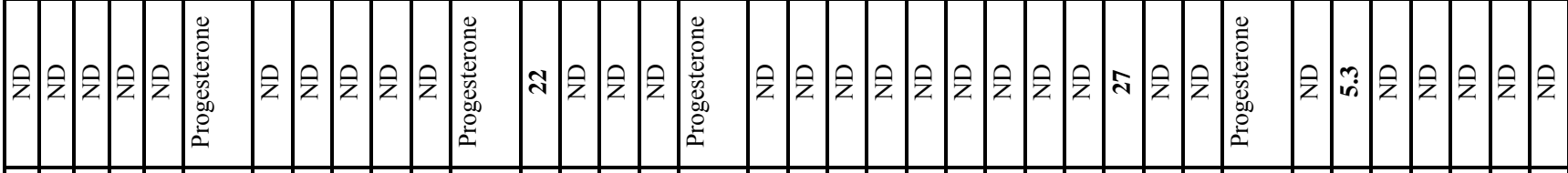

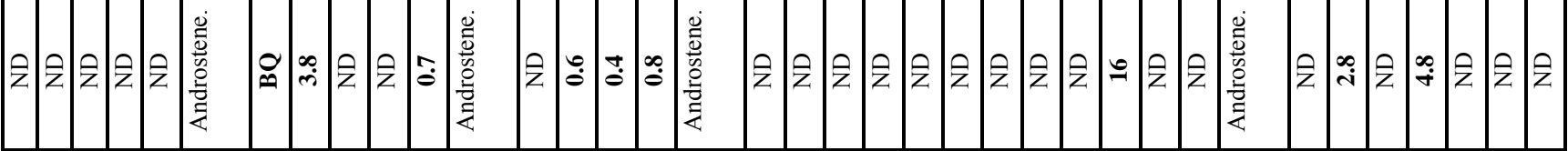

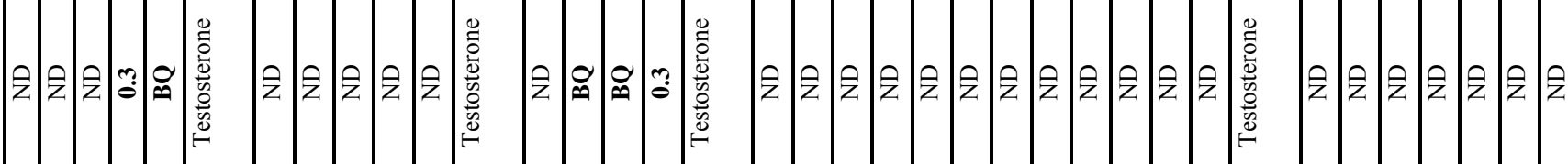

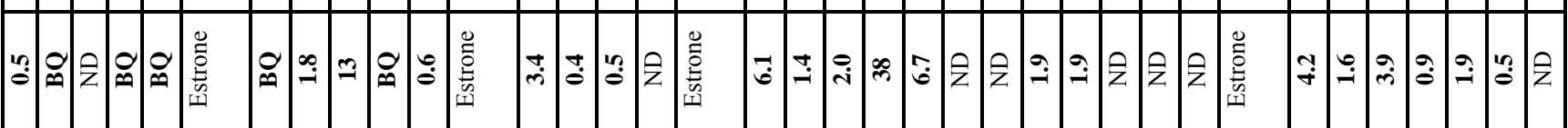

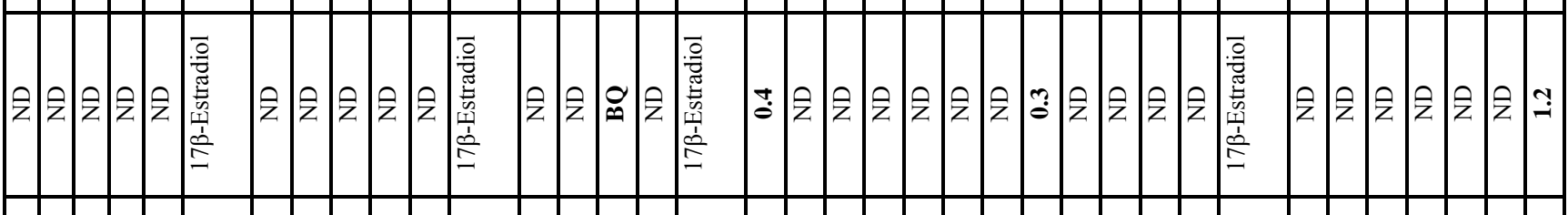

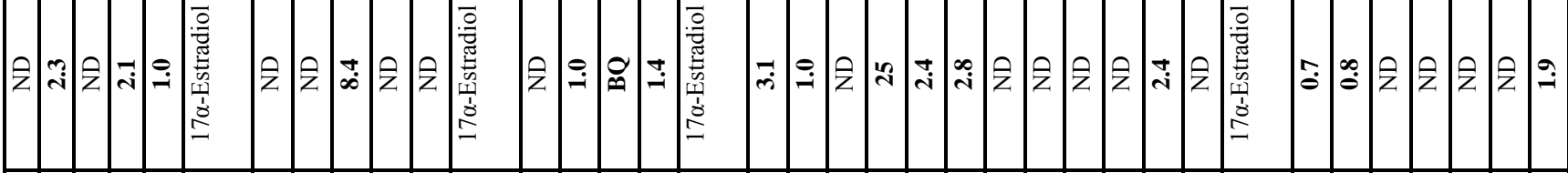

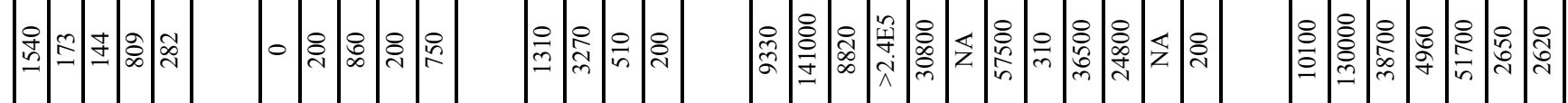

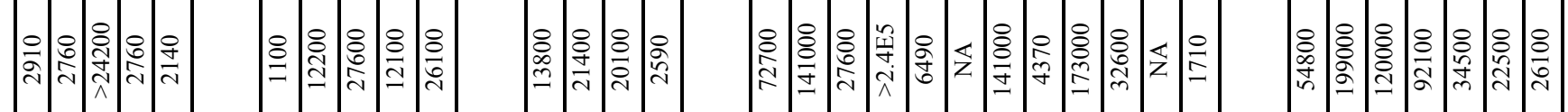

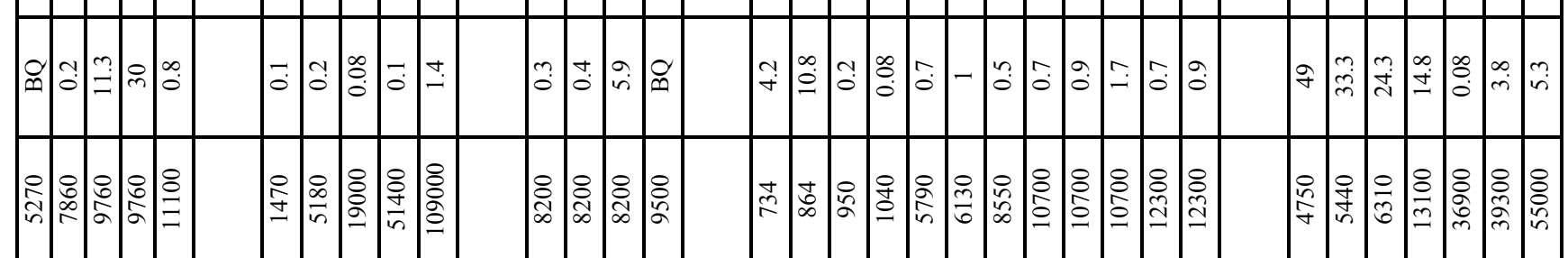

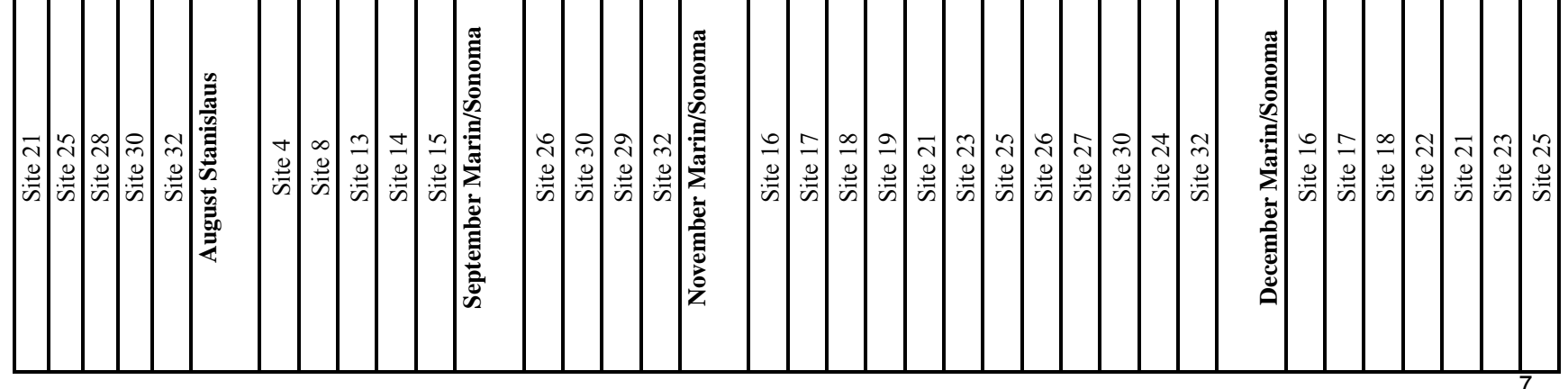


云

zิ

そิ

そิ

○

そ 2 そ

鱼

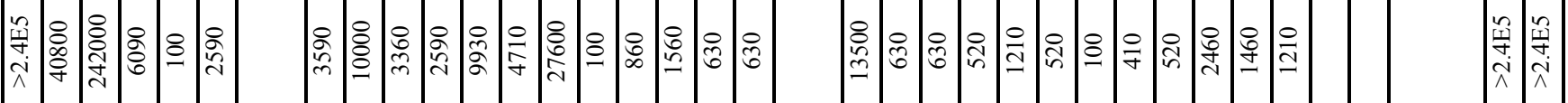

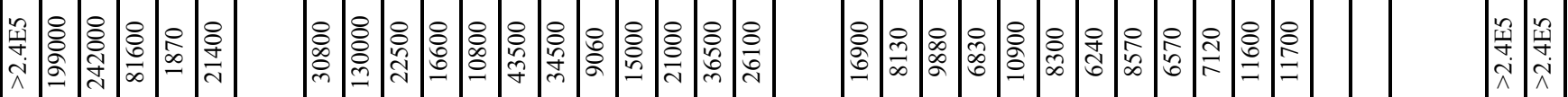

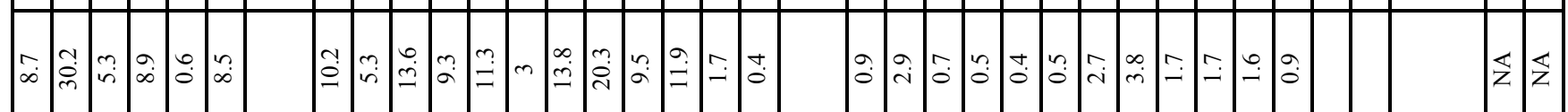

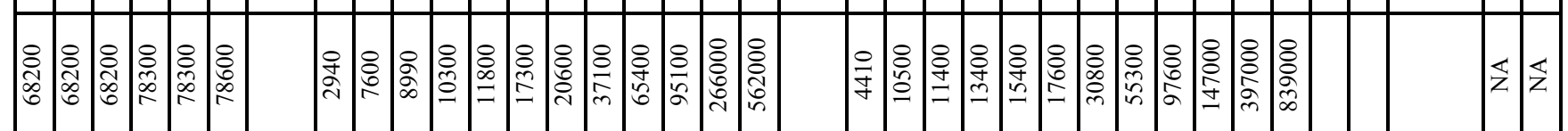

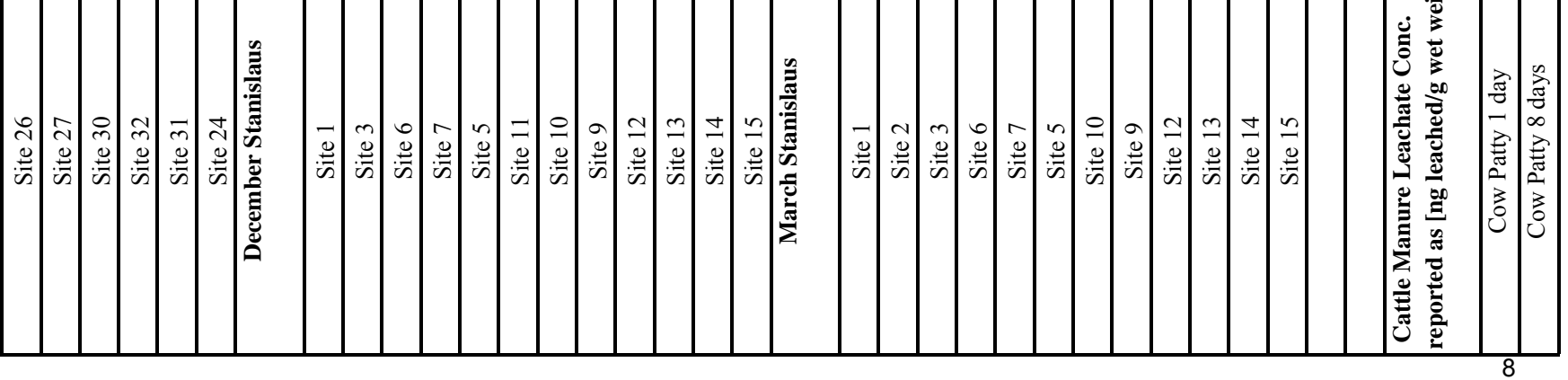




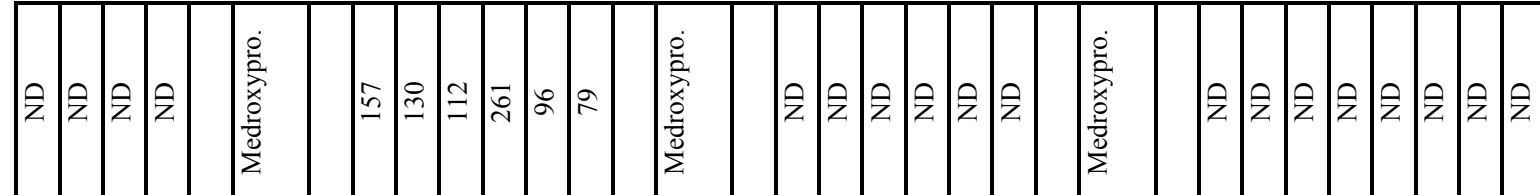

z人三

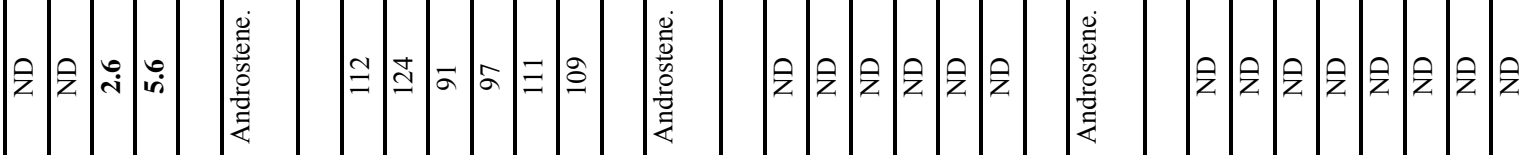

之े

乎

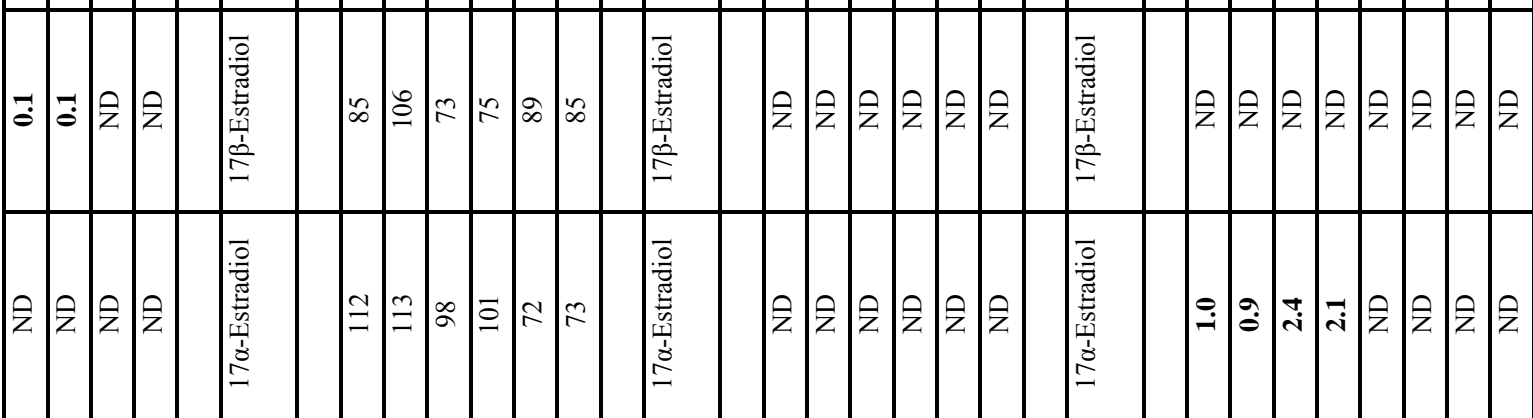

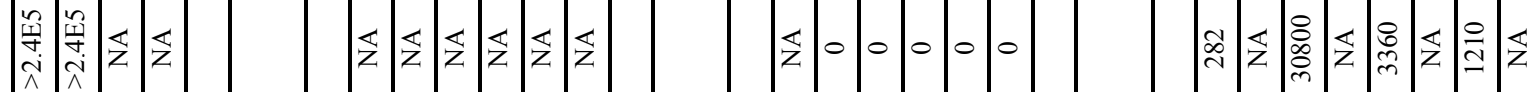

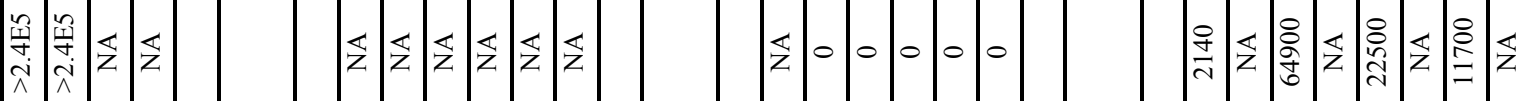

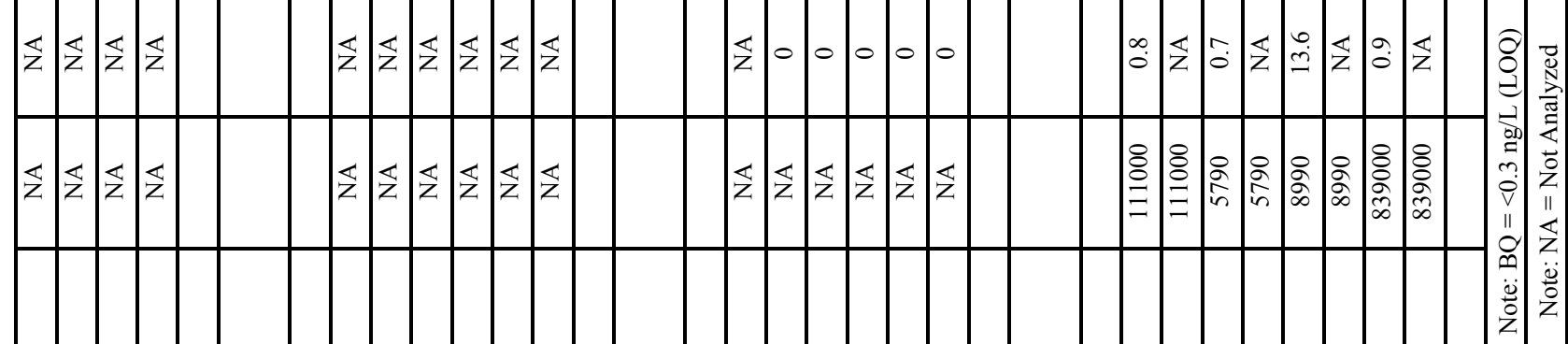

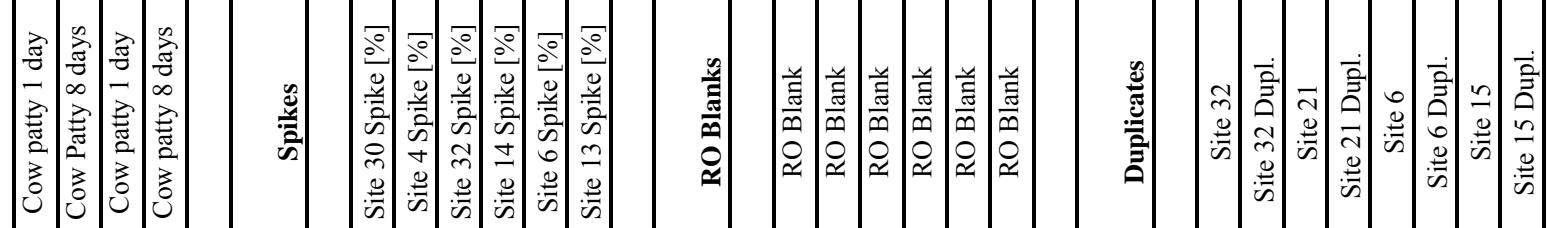


Table S3: Mass discharges for androgens and progesterone as well as the number of cows needed to explain the observed discharges.

Kolodziej et al

"Rangeland grazing as a source of steroid hormones to surface waters"

\begin{tabular}{|c|c|c|c|c|c|}
\hline \multirow[b]{2}{*}{ Location } & \multirow[b]{2}{*}{ Date } & \multicolumn{2}{|c|}{ Mass Discharges [ug/day] } & \multicolumn{2}{|c|}{ Number of Cows [\#] ${ }^{\text {a }}$} \\
\hline & & Androgens & Progesterone & Androgens & Progesterone \\
\hline Site 2 & $4 / 22 / 2005$ & 330 & 0 & 0.3 & 0 \\
\hline Site 3 & $4 / 22 / 2005$ & 350 & 0 & 0.3 & 0 \\
\hline Site 10 & $4 / 22 / 2005$ & 950 & 0 & 0.9 & 0 \\
\hline Site 30 & $6 / 23 / 2005$ & 2900 & 0 & 2.7 & 0 \\
\hline Site 32 & $6 / 23 / 2005$ & 1100 & 0 & 1 & 0 \\
\hline Site 4 & $8 / 31 / 2005$ & 150 & 0 & 0.1 & 0 \\
\hline Site 8 & $8 / 31 / 2005$ & 20000 & 0 & 18 & 0 \\
\hline Site 15 & $8 / 31 / 2005$ & 76000 & 0 & 69 & 0 \\
\hline Site 26 & $9 / 8 / 2005$ & 0 & 180000 & 0 & 15 \\
\hline Site 29 & $9 / 8 / 2005$ & 4100 & 0 & 3.7 & 0 \\
\hline Site 30 & $9 / 8 / 2005$ & 5700 & 0 & 5.2 & 0 \\
\hline Site 32 & 9/8/2005 & 10000 & 0 & 9.5 & 0 \\
\hline Site 30 & $11 / 8 / 2005$ & 170000 & 290000 & 156 & 24 \\
\hline Site 17 & $12 / 2 / 2005$ & 15000 & 29000 & 14 & 2.4 \\
\hline Site 22 & $12 / 2 / 2005$ & 63000 & 0 & 57 & 0 \\
\hline Site 30 & $12 / 2 / 2005$ & 3000000 & 1400000 & 2730 & 114 \\
\hline Site 1 & $12 / 29 / 2005$ & 6800 & 0 & 6.1 & 0 \\
\hline Site 10 & $12 / 29 / 2005$ & 50000 & 0 & 45 & 0 \\
\hline Site 3 & $3 / 9 / 2006$ & 1100 & 0 & 1 & 0 \\
\hline Site 5 & $3 / 9 / 2005$ & 1800 & 0 & 1.6 & 0 \\
\hline Site 6 & $3 / 9 / 2005$ & 1300 & 0 & 1.2 & 0 \\
\hline Site 12 & $3 / 9 / 2005$ & 9800 & 0 & 8.9 & 0 \\
\hline
\end{tabular}

${ }^{a}$ Steroid excretion data from Lange et al . (2002). 